\title{
Resveratrol and Malignancies
}

\author{
Rodica P. Bunaciu $\cdot$ Andrew Yen
}

Published online: 10 March 2015

(C) Springer International Publishing AG 2015

\begin{abstract}
Carcinogenesis is a multifactorial process, frequently encompassing three stages: initiation, promotion, and progression. It is characterized by multiple deviations from normal both at the cell and organism levels. Although most people have a small number of cells that present deviations from normal, most of those cells will not cause cancer. However, some will. What tips the balance between normal and abnormal is the subject of intense scientific research as well as unfounded speculations. Chronic inflammation is one of the risk factors for cancer. Resveratrol is consumed by the population as a dietary supplement in the hope of decreasing the risk of inflammation and cancer and other chronic diseases such as diabetes and vascular diseases. There is a discrepancy between the doses used in the animal studies showing that resveratrol decreases all three stages of carcinogenesis and the doses ingested by the population either as supplements or in the diet. While there is health benefit from using high resveratrol doses, it might be also of practical and scientific benefit to focus future effort in understanding the effects of normal dietary resveratrol levels.
\end{abstract}

Keywords Resveratrol $\cdot 3,4^{\prime}, 5$-Trihydroxy-trans-stilbene, 5-[(1E)-2-(4-hydroxyphenyl) ethenyl]-1,3-benzenediol . Stilbene $\cdot$ Cancer $\cdot$ Human

This article is part of the Topical Collection on Cancer Chemoprevention

R. P. Bunaciu

Department of Biomedical Sciences, TB-013 VRT,

Cornell University, Ithaca, NY 14853, USA

e-mail: rpb78@cornell.edu

A. Yen $(\bowtie)$

Department of Biomedical Sciences, T4-008 VRT,

Cornell University, Ithaca, NY 14853, USA

e-mail: ay13@cornell.edu

\section{Introduction}

According to the $\mathrm{CDC}$, regarding incidence, the top 10 cancer sites (2007-2011) in males in the USA are the following: prostate, lung and bronchus, colon and rectum, urinary bladder, melanomas of the skin, non-Hodgkin lymphoma, kidney and renal pelvis, oral cavity and pharynx, leukemias, and pancreas. The top 10 cancer sites (2007-2011) in females in the USA are the following: breast, lung and bronchus, colon and rectum, corpus and uterus not otherwise specified (NOS), thyroid, non-Hodgkin lymphoma, melanomas of the skin, ovary, kidney and renal pelvis, and pancreas. In children $(0-19$ years old), the invasive cancer with the highest incidence rates are acute lymphocytic leukemia, brain and other nervous system, non-Hodgkin lymphoma, Hodgkin lymphoma, soft tissue, bones and joints, acute myeloid leukemia, kidney, and renal pelvis. Some cancers, although their incidence rates do not reach the top 10 , have death rates that are among the top 10 : liver and intrahepatic bile duct, urinary bladder, and esophagus in men and leukemias, brain and other nervous system, and liver and intrahepatic bile duct in women.

Resveratrol is consumed by the population as chemopreventive agent. From the seminal paper published in Science in 1997 [1] till now, more than 7000 scientific papers were published on resveratrol (http://www.ncbi.nlm.nih.gov/pubmed/? term=resveratrol, as searched on $1 / 20 / 2015$ ). The initial enthusiasm evolved into partial disappointment due to the awareness that resveratrol affects most of the known biochemical and signaling pathways $[2,3]$ and due to the high doses needed to elicit those cellular responses $[2,4 \bullet \bullet]$. This review seeks to summarize the effects of resveratrol regarding the main human cancers and to propose a few new directions for resveratrol chemoprevention.

\section{Resveratrol}

Resveratrol is a polyphenol also known under the synonym name 3,4',5-trihydroxy-trans-stilbene, or 5-[(1E)-2-(4- 
hydroxyphenyl) ethenyl]-1,3-benzenediol. It is a dietary naturally occurring phytoalexin. Its main dietary sources in human diet are the skin and seeds of grapes, peanuts, and mulberries [1]. It is also very abundant in the root of Polygonum cuspidatum [5••]. In an excellent review, $\mathrm{Ma}$ and $\mathrm{Hu}$ assess essential facts about resveratrol's fate across the gastrointestinal tract $[4 \bullet \bullet$. They review the main publications showing that piceid, the $3-\beta$-glucoside of resveratrol, the most abundant form of resveratrol in nature, is cleaved by intestinal flora to resveratrol and a sugar moiety. They also show low aqueous solubility of resveratrol and low bioavailability; however, the low bioavailability is not due exclusively to low solubility [40. ]. It is important to point out that although the bioavailability is low, resveratrol is nevertheless bioavailable following oral administration [2]. Resveratrol crosses the intestinal apical membrane due to its lipophilic nature, and in Caco-2 cells, a transporter, SGLT1, was reported as being important especially for the transport of piceid which is hydrolysed to resveratrol in the epithelial cell [4.•]. The low oral bioavailability of resveratrol is thought to be due to phase II metabolism in the enterocyte [4••]. However, there are expert opinions that this is not an impediment. The biological effects of resveratrol are also due to its metabolites [2]. MRP2 on apical side of enterocytes (transporting back into the intestinal lumen) and MRP3 on the basolateral side (transporting to the portal vein and lymph duct) are thought to be active in excreting the metabolites of resveratrol $[4 \bullet \cdot]$. The apical efflux from enterocytes might be the main cause of low bioavailability. The enterohepatic recycling of resveratrol is also important for bioavailability, but less studied. Resveratrol is present in serum and urine predominantly as glucuronide and sulfate conjugates [6].

\section{Cancer, Inflammation, and Resveratrol}

Although the causes of cancer are multiple and not easy to classify, chronic inflammation is recognized as a leading risk factor. Chronic inflammation is a significant public health concern in Western populations. There are multiple causes and molecular mechanisms of chronic inflammation. There are genetic and metabolic factors but also dietary and environmental factors. Many people use resveratrol in order to prevent chronic inflammation and associated pathologies, such as cardiovascular diseases, metabolic syndrome, and cancer. In fact, the first discovered biological effect of resveratrol was its anti-inflammatory effect by modulating arachidonate metabolism [7] and inhibiting cyclooxygenase [1]. A plant rich in resveratrol, $P$. cuspidatum, is highly used in Chinese medicine to treat inflammation and cancer as well as metabolic diseases [5••], and it is the main source of resveratrol for commercially available supplements. Resveratrol (in high doses) was rigorously demonstrated to hinder cancer initiation, promotion, and progression in 1997 [1].

\section{Is there Evidence that Resveratrol is Relevant Against Human Cancers?}

After decades of research on resveratrol, and high hopes for its antitumor effects, there is still not a clear understanding of its effectiveness. Below, we present just a few examples of studies on resveratrol for the main human cancers. Those examples show the broad range of cancer types in which resveratrol was tested, encompassing most of the prevalent cancers.

\section{Colon and Rectum Cancers}

Most clinical trials investigating the anticancer effects of resveratrol were in colon cancer.

In trial NCT00256334, resveratrol-containing freeze-dried grape powder (low dosages of resveratrol in combination with other bioactive components) can inhibit the Wnt pathway in vivo [8].

In NCT00578396 (phase 1), primary cell lines generated from resected colorectal tumor specimens were treated with mitomycin $\mathrm{C}$ and resveratrol and had greater upregulation of p21(WAF1/CIP1) (which inhibits the cell cycle at G0/G1 and G2/M phases) compared with the cells treated with either agent alone [9].

There are also two other clinical trials with less information available so far: NCT00920803 (phase 1) and NCT00433576 that assess resveratrol in treating patients with colorectal cancer that can be removed by surgery (phase 1). In another clinical study performed in the UK, 20 patients with histologically confirmed colorectal cancer consumed eight daily doses of resveratrol at 0.5 or $1.0 \mathrm{~g}$ before surgical resection. Resveratrol and its metabolites, resveratrol-3-O-glucuronide, resveratrol-4'-O-glucuronide, resveratrol-3- $O$-sulfate, resveratrol-4'$O$-sulfate, resveratrol sulfate glucuronide, and resveratrol disulfate, were present in colorectal resection tissue. Resveratrol reduced cell proliferation by $5 \%$ [10].

Liver and Intrahepatic Bile Duct and Gastrointestinal Cancers

There have been a couple of GI clinical trials: ACTR N12612001135808 and NCT01476592. In nonalcoholic fatty liver disease (NAFLD) patients, $3 \mathrm{~g} /$ day resveratrol for 8 weeks increased levels of alanine and aspartate aminotransferases, markers of hepatic stress [11]. NAFLD is a risk factor for hepatocellular carcinoma. NCT01476592 is an active clinical trial assessing resveratrol's effects on notch-1 signaling in subjects with low-grade gastrointestinal tumors. In a phase I randomized, double-blind pilot study performed in the UK in patients with hepatic metastases, receiving $5.0 \mathrm{~g}$ micronized 
resveratrol (SRT501) daily for 14 days, it was found that this regimen was well tolerated and micronized SRT501 led to higher bioavailability than resveratrol. This regimen led to a significant increase in cleaved caspase-3, a marker of apoptosis, in malignant hepatic tissue [12].

\section{Leukemias}

The potential to use resveratrol in chronic myelogenous leukemia and $\mathrm{Ph}+$ acute lymphoblastic leukemia as reviewed by Vakana and Platanias [13] showed that resveratrol activates the heterotrimeric AMP-activated protein kinase (AMPK) complex and JNK-dependent p62/SQSTM1. They also pointed out Puissant and collaborators' important finding that resveratrol has the potential to induce apoptosis in some imatinib-resistant chronic myelogenous leukemia cells [14]. In the initial report of resveratrol's antitumor activity, Pezzuto and collaborators used HL-60 myelo-monocytic leukemia cells to show that resveratrol caused growth arrest and induction of differentiation (assessed by respiratory burst) [1]. Aggarwal and collaborators showed that resveratrol also suppressed colony-forming cell proliferation of fresh AML marrow cells from patients with newly diagnosed AML in a dosedependent fashion [15].

\section{Lymphoma}

A population-based genetic association study showed correlation between mutations in AMPK subunit genes and the risk of non-Hodgkin lymphoma [16]. As resveratrol activates AMPK [13], there is hope that it has chemopreventive effects for lymphoma. In Hodgkin lymphoma (HL)-derived L-428 cells, resveratrol induces apoptosis by SIRT1 inhibition and FOXO3a hyperacetylation [17].

\section{Multiple Myeloma}

NCT00920556 was a terminated phase 2 study on multiple myeloma with $5 \mathrm{~g}$ /day SRT501 in 21-day cycles. It was a study of SRT501 (resveratrol) with bortezomib for patients with relapsed and or refractory multiple myeloma. It showed that the disease stabilization caused by bortezomib alone can prevent renal failure [18].

\section{Brain and Other Nervous System Cancers}

In patient isolated glioma stem cells, resveratrol activates the p53/p21 pathway and suppresses Nanog [19••]. In medulloblastoma cell line, resveratrol with 5-Aza-dC inhibits metabolic activity [20].

\section{Soft Tissue Cancers}

Resveratrol inhibits rhabdomyosarcoma cell proliferation by causing S/G2 arrest and cyclin B downregulation, in a human embryonal rhabdomyosarcoma model [21]. Resveratrol together with clofarabine inhibits mesothelioma MSTO-211H cell growth, Nrf2 activation, and its target gene heme oxygenase-1 (HO-1) expression [22].

Bone and Joint Cancers

Using a bone cancer pain model, resveratrol was recently suggested to have analgesic effects by inhibiting the spinal glial activation and decreasing CX3CR1 [23].

\section{Thyroid Cancers}

Resveratrol activates Notch1 signaling, induces differentiation (TTF1 and Pax8), and suppresses cell growth in anaplastic thyroid carcinoma [24].

\section{Pancreatic Cancer}

In pancreatic cancer stem cells, resveratrol inhibited selfrenewal capacity, migration and invasion, Nanog, SOX-2, cMyc, Oct4 and ABCG2 (markers of pluripotency), Zeb-1, and Slug and Snail (markers of epithelial-mesenchymal transition) [25].

\section{Prostate Cancer}

NCT01317199 is a clinical trial led by Johns Hopkins University which is estimated to be completed this year. It is a phase 1 and 2 clinical trial aiming to assess effects of two doses of "Muscadine Plus Grape Skin Extract" capsules on rising prostate-specific antigen levels in men following initial therapy for prostate cancer.

\section{Breast Cancer}

NCT01370889 was completed. In overweight and obese postmenopausal women, $1 \mathrm{~g}$ /day resveratrol showed favorable effects on estrogen metabolism and a $10 \%$ increase in the concentrations of sex steroid hormone-binding globulin, thus reducing the breast cancer risk factors in this population [26]. In MCF-10A cells, resveratrol prevented $17 \beta$-estradiolinduced decrease in SOD3, NQO1, and Nrf2 mRNA levels [27].

The relationship between estrogen levels, persistent organic pollutants, and resveratrol as modulators of cancer risk deserves special attention. In animal models, it has been shown that gestational exposure to the xenobiotic 2,3,7,8tetrachlorodibenzo- $p$-dioxin induces BRCA-1 promoter 
hypermethylation and reduces BRCA-1 expression in mammary tissue of the offspring [28]. Those effects have been shown to be prevented by resveratrol in the MCF-7 cell model [28]. Moreover, aryl hydrocarbon receptor, also known as dioxin receptor, $(\mathrm{AhR})$ has an important role in modulating the immune system [29-33]. Resveratrol was reported to inhibit breast cancer metastasis by inactivating the tumorevoked regulatory B cells [34].

\section{Remarks and New Directions}

\section{AhR and NRF2 as Inflammation-Environment-Diet Molecular Cross-roads}

The main reason people take resveratrol is to prevent inflammation, a physiological response to an environmental or internal insult. The potential link between AhR as a modulator of immunity (inflammation) and response to xenobiotics on one hand and resveratrol activities on the other hand is intriguing. Moreover, it is interesting to mention that the effect of resveratrol is frequently associated with upregulation or activation of Nrf2 [35-37]. Furthermore, resveratrol upregulates phase II metabolism enzyme (such as HO-1, NQO1) expression by increasing the transcriptional activity of Nrf2. Those phase II metabolism enzymes are also transcriptionally controlled by $\mathrm{AhR}$ via XRE in their promoters [38]. Moreover, many people taking resveratrol also take AhR-inducing agents, such as valproic acid as mood stabilizer, chemotherapy, and Ginkgo biloba. Additionally, we are all exposed to various AhRactivating environmental pollutants. There are also dietary AhR antagonists such as genistein, kaempferol, or EGCG. $\mathrm{AhR}$ is an important protein orchestrating stacking (resultant effect of more than one dietary supplement), drug-supplement or pollutant-supplement effects. Important players in inflammation are monocytes and macrophages and their vasculature interactions. A relatively novel AhR synthetic agonist, VAF347, is a low molecular weight, cell permeable compound that causes a number of anti-inflammatory responses in vitro and in vivo [39]. For example, it inhibits the development of $\mathrm{CD} 14^{+} \mathrm{CD} 11 \mathrm{~b}^{+}$monocytes from granulo-monocytic (GM stage) precursors [40]. Resveratrol is reported to decrease inflammation and improve vascular parameters. It would be of interest to elucidate in greater detail the contribution of $\mathrm{AhR}$ to resveratrol's effects and the collaboration of AhR with Nrf2, especially in the maintenance of homeostasis. It is an attractive hypothesis that low (dietary) to moderate (supplements) doses of resveratrol assist Nrf2 and AhR in maintaining homeostasis against environmental or inflammatory insults that otherwise might be tumor initiators. Due to the long effect time and multiparameter nature (including many confounding factors), it will be difficult to test this hypothesis.
Resveratrol in Other Clinical Trials with Relevance for Cancer Prevention

Although not directly investigating antitumor effects, some resveratrol studies are relevant to its possible anticancer effects or to improving the well-being of cancer patients or survivors. The NCT01354977 clinical trial aims to assess the effect of resveratrol on age-related inflammation in humans, and although it does not directly assess cancer parameters, it asses a recognized risk factor. NCT01043939 assessed the effect of resveratrol-rich grape juice in childhood cancer survivors. In another study, $200 \mathrm{mg} /$ day resveratrol intake for 26 weeks in healthy older adults resulted in reductions in body weight and BMI and decreases in systolic and diastolic blood pressure associated with improvements in the integrity and functionality of the hippocampus [41]. In conclusion, there is encouraging data, both for potential antitumor effects as well as improving the well-being of patients and the general population.

A large number of clinical trials investigate the healthpromoting and therapeutic effects of resveratrol; however, it is noteworthy mentioning that the doses used in most of the clinical trials are much higher (at least 10 times higher) than the dose one person would ingest in the diet or by taking the label recommended dose of commercially available supplements. Moreover, taken in the diet, resveratrol is likely to have other nutrient-resveratrol interactions compared to being taken as a supplement.

\section{Conclusions}

There is a grey zone in the public perception and perhaps at a certain degree even in the scientific literature, between the high-dose pharmacological effects of resveratrol (including its structural or functional analogues) and the effects of dietary resveratrol. We believe that both have their merits, but are clearly distinct. Some clinical studies investigate the effects of very high doses of resveratrol, aiming to assess its therapeutic role. Perhaps, prospective studies assessing the intake of resveratrol supplements and consumption of foods rich in resveratrol on the one hand and assessing cancer incidence on the other hand could clarify the association between resveratrol intake and cancer incidence and thus its chemopreventive role.

Regarding its molecular role, it appears that resveratrol, at least in high concentrations as used in most studies, affects most signaling and metabolic pathways. The shift in the perception that resveratrol directly regulates Sirt1 to the current accent on cAMP signaling $[13,42 \bullet \cdot]$ deserves reflection and points again to the need of studying the effect of lower doses. Historically, resveratrol was intensely studied in the aging process as a direct activator of SIRT1. Its presence in red wine 
propelled it to headlines in newspapers. The controversy about the assay measuring the SIRT1 activity and the involvement of SIRT1 in aging led to a decrease in the enthusiasm level for resveratrol studies in general $[43,44]$. This piece of modern science history points to the need for moderation in our interpretations of the scientific findings, avoiding both passionate desire of finding elixir vitae, "elixir of life", and hasten dismissal of any health benefits of one natural compound once our utopian dreams were not met.

We regret it was not possible because of space limitations to cite all of the important work that has been reported in this broad and active field and apologize for omissions.

Acknowledgments We gratefully acknowledge the partial support from the National Institutes of Health (NIH) CA033505 (Yen), CA152870 (Yen), 1U54 CA143876 (Shuler), and New York State Stem Cell Science (NYSTEM) (Yen).

\section{Compliance with Ethics Guidelines}

Conflict of Interest Rodica P Bunaciu and Andrew Yen declare that they have no conflict of interest.

Human and Animal Rights and Informed Consent This article does not contain any studies with human or animal subjects performed by any of the authors.

\section{References}

Papers of particular interest, published recently, have been highlighted as:

•- Of major importance

1. Jang M, Cai L, Udeani GO, Slowing KV, Thomas CF, Beecher CW, et al. Cancer chemopreventive activity of resveratrol, a natural product derived from grapes. Science. 1997;275:218-20.

2. Pezzuto JM. The phenomenon of resveratrol: redefining the virtues of promiscuity. Ann N Y Acad Sci. 2011;1215:123-30.

3. Baur JA, Pearson KJ, Price NL, Jamieson HA, Lerin C, Kalra A, et al. Resveratrol improves health and survival of mice on a highcalorie diet. Nature. 2006;444:337-42.

4.• Ma Y, Hu M. Metabolism and transport of anticancer and antiinflammarory phytochemicals across the gastrointestinal tract. In: Kong AN, editor. Inflammation, oxidative stress, and cancer: dietary approaches for cancer prevention. Boca Raton: Taylor \& Francis Group, LLT; 2014. p. 135-69. This is a seminal review and presents iformation absolutely essential for understanding the fate of a phytochemical (resveratrol included) after ingestion.

5.• Peng W, Qin R, Li X, Zhou H. Botany, phytochemistry, pharmacology, and potential application of Polygonum cuspidatum Sieb.et Zucc.: a review. J Ethnopharmacol. 2013;148:729-45. An important review of a plant highly used by the population.

6. Goldberg DM, Yan J, Soleas GJ. Absorption of three wine-related polyphenols in three different matrices by healthy subjects. Clin Biochem. 2003;36:79-87.

7. Kimura Y, Okuda H, Arichi S. Effects of stilbenes on arachidonate metabolism in leukocytes. Biochim Biophys Acta. $1985 ; 834: 275-8$.
8. Nguyen AV, Martinez M, Stamos MJ, Moyer MP, Planutis K, Hope $\mathrm{C}$, et al. Results of a phase I pilot clinical trial examining the effect of plant-derived resveratrol and grape powder on Wnt pathway target gene expression in colonic mucosa and colon cancer. Cancer Manag Res. 2009;1:25-37.

9. Ali I, Braun DP. Resveratrol enhances mitomycin C-mediated suppression of human colorectal cancer cell proliferation by upregulation of p21WAF1/CIP1. Anticancer Res. 2014;34:5439-46.

10. Patel KR, Brown VA, Jones DJ, Britton RG, Hemingway D, Miller AS, et al. Clinical pharmacology of resveratrol and its metabolites in colorectal cancer patients. Cancer Res. 2010;70:7392-9.

11. Chachay VS, Macdonald GA, Martin JH, Whitehead JP, O'MooreSullivan TM, Lee P, et al. Resveratrol does not benefit patients with nonalcoholic fatty liver disease. Clin Gastroenterol Hepatol Off Clin Pract J Am Gastroenterol Assoc. 2014;12(12):2092-103.

12. Howells LM, Berry DP, Elliott PJ, Jacobson EW, Hoffmann E, Hegarty B, et al. Phase I randomized, double-blind pilot study of micronized resveratrol (SRT501) in patients with hepatic metastases-safety, pharmacokinetics, and pharmacodynamics. Cancer Prev Res (Phila). 2011;4:1419-25.

13. Vakana E, Platanias LC. AMPK in BCR-ABL expressing leukemias. Regulatory effects and therapeutic implications. Oncotarget. 2011;2:1322-8.

14. Puissant A, Grosso S, Jacquel A, Belhacene N, Colosetti P, Cassuto $\mathrm{JP}$, et al. Imatinib mesylate-resistant human chronic myelogenous leukemia cell lines exhibit high sensitivity to the phytoalexin resveratrol. FASEB J Off Publ Fed Am Soc Exp Biol. 2008;22:1894 904.

15. Estrov Z, Shishodia S, Faderl S, Harris D, Van Q, Kantarjian HM, et al. Resveratrol blocks interleukin-1beta-induced activation of the nuclear transcription factor NF-kappaB, inhibits proliferation, causes S-phase arrest, and induces apoptosis of acute myeloid leukemia cells. Blood. 2003;102:987-95.

16. Hoffman AE, Demanelis K, Fu A, Zheng T, Zhu Y. Association of AMP-activated protein kinase with risk and progression of nonHodgkin lymphoma. Cancer Epidemiol Biomark Prev. 2013;22: 736-44.

17. Frazzi R, Valli R, Tamagnini I, Casali B, Latruffe N, Merli F. Resveratrol-mediated apoptosis of Hodgkin lymphoma cells involves SIRT1 inhibition and FOXO3a hyperacetylation. Int $\mathrm{J}$ Cancer. 2013;132:1013-21.

18. Popat R, Plesner T, Davies F, Cook G, Cook M, Elliott P, et al. A phase 2 study of SRT501 (resveratrol) with bortezomib for patients with relapsed and or refractory multiple myeloma. Br J Haematol. 2013;160:714-7.

19.• Sato A, Okada M, Shibuya K, Watanabe E, Seino S, Suzuki K, et al. Resveratrol promotes proteasome-dependent degradation of Nanog via p53 activation and induces differentiation of glioma stem cells. Stem Cell Res. 2013;11:601-10. The paper presents a mechanism of action of resveratrol involving downregulation of the stem cell marker Nanog.

20. Patties I, Kortmann RD, Glasow A. Inhibitory effects of epigenetic modulators and differentiation inducers on human medulloblastoma cell lines. J Exp Clin Cancer Res CR. 2013;32:27.

21. Chow AW, Murillo G, Yu C, van Breemen RB, Boddie AW, Pezzuto JM, et al. Resveratrol inhibits rhabdomyosarcoma cell proliferation. Eur J Cancer Prev. 2005;14:351-6.

22. Lee YJ, Im JH, Lee DM, Park JS, Won SY, Cho MK, et al. Synergistic inhibition of mesothelioma cell growth by the combination of clofarabine and resveratrol involves Nrf2 downregulation. BMB Rep. 2012;45:647-52.

23. Cheng W, Zhao Y, Liu H, Fan Q, Lu FF, Li J, et al. Resveratrol attenuates bone cancer pain through the inhibition of spinal glial activation and CX3CR1 upregulation. Fundam Clin Pharmacol. 2014;28(6):661-70. 
24. Yu XM, Jaskula-Sztul R, Ahmed K, Harrison AD, Kunnimalaiyaan $\mathrm{M}$, Chen $\mathrm{H}$. Resveratrol induces differentiation markers expression in anaplastic thyroid carcinoma via activation of Notch1 signaling and suppresses cell growth. Mol Cancer Ther. 2013;12:1276-87.

25. Shankar S, Nall D, Tang SN, Meeker D, Passarini J, Sharma J, et al. Resveratrol inhibits pancreatic cancer stem cell characteristics in human and KrasG12D transgenic mice by inhibiting pluripotency maintaining factors and epithelial-mesenchymal transition. PLoS One. 2011;6:e16530.

26. Chow HH, Garland LL, Heckman-Stoddard BM, Hsu CH, Butler VD, Cordova CA, et al. A pilot clinical study of resveratrol in postmenopausal women with high body mass index: effects on systemic sex steroid hormones. J Transl Med. 2014;12:223.

27. Chatterjee A, Ronghe A, Singh B, Bhat NK, Chen J, Bhat HK. Natural antioxidants exhibit chemopreventive characteristics through the regulation of $\mathrm{CNC}$ b-Zip transcription factors in estrogen-induced breast carcinogenesis. J Biochem Mol Toxicol. 2014;28(12):529-38.

28. Papoutsis AJ, Selmin OI, Borg JL, Romagnolo DF. Gestational exposure to the AhR agonist 2,3,7,8-tetrachlorodibenzo-p-dioxin induces BRCA-1 promoter hypermethylation and reduces BRCA-1 expression in mammary tissue of rat offspring: Preventive effects of resveratrol. Molecular carcinogenesis. 2013. doi:10.1002/mc.22095.

29. Sherr DH, Monti S. The role of the aryl hydrocarbon receptor in normal and malignant B cell development. Semin Immunopathol. 2013;35:705-16.

30. Stockinger B, Hirota K, Duarte J, Veldhoen M. External influences on the immune system via activation of the aryl hydrocarbon receptor. Semin Immunol. 2011;23:99-105.

31. Veldhoen M, Duarte JH. The aryl hydrocarbon receptor: fine-tuning the immune-response. Curr Opin Immunol. 2010;22:747-52.

32. Marshall NB, Kerkvliet NI. Dioxin and immune regulation: emerging role of aryl hydrocarbon receptor in the generation of regulatory T cells. Ann N Y Acad Sci. 2010;1183:25-37.

33. Stockinger B. Beyond toxicity: aryl hydrocarbon receptor-mediated functions in the immune system. J Biol. 2009;8:61.

34. Lee-Chang C, Bodogai M, Martin-Montalvo A, Wejksza K, Sanghvi M, Moaddel R, et al. Inhibition of breast cancer metastasis by resveratrol-mediated inactivation of tumor-evoked regulatory $\mathrm{B}$ cells. J Immunol. 2013;191:4141-51.
35. Cheng AS, Cheng YH, Chiou CH, Chang TL. Resveratrol upregulates $\mathrm{Nrf} 2$ expression to attenuate methylglyoxal-induced insulin resistance in Hep G2 cells. J Agric Food Chem. 2012;60: 9180-7.

36. Rubiolo JA, Mithieux G, Vega FV. Resveratrol protects primary rat hepatocytes against oxidative stress damage: activation of the $\mathrm{Nrf} 2$ transcription factor and augmented activities of antioxidant enzymes. Eur J Pharmacol. 2008;591:66-72.

37. Ungvari Z, Bagi Z, Feher A, Recchia FA, Sonntag WE, Pearson K, et al. Resveratrol confers endothelial protection via activation of the antioxidant transcription factor Nrf2. Am J Physiol Heart Circ Physiol. 2010;299:H18-24.

38. Shu L, Zhang C, Kong AN. Overview of common dietary phytochemicals possesing antioxidant properties through Nrf2. In: Kong $\mathrm{AN}$, editor. Inflammation, oxidative stress, and cancer: dietary approaches for cancer prevention. Boca Raton: Taylor \& Francis Group, LLT; 2014. p. 55-77.

39. Baba N, Rubio M, Kenins L, Regairaz C, Woisetschlager M, Carballido JM, et al. The aryl hydrocarbon receptor (AhR) ligand VAF347 selectively acts on monocytes and naive CD4(+) Th cells to promote the development of IL-22-secreting Th cells. Hum Immunol. 2012;73:795-800.

40. Platzer B, Richter S, Kneidinger D, Waltenberger D, Woisetschlager M, Strobl H. Aryl hydrocarbon receptor activation inhibits in vitro differentiation of human monocytes and Langerhans dendritic cells. J Immunol. 2009;183:66-74.

41. Witte AV, Kerti L, Margulies DS, Floel A. Effects of resveratrol on memory performance, hippocampal functional connectivity, and glucose metabolism in healthy older adults. J Neurosci Off J Soc Neurosci. 2014;34:7862-70.

42.• Park SJ, Ahmad F, Philp A, Baar K, Williams T, Luo H, et al. Resveratrol ameliorates aging-related metabolic phenotypes by inhibiting cAMP phosphodiesterases. Cell. 2012;148:421-33. The paper presents a paradigm shift in the resveratrol mechanism of action.

43. Ledford H. Ageing: much ado about ageing. Nature. 2010;464: 480-1.

44. Couzin-Frankel J. Genetics. Aging genes: the sirtuin story unravels. Science. 2011;334:1194-8. 